\title{
Candidate exoplanet host HD 131399A: a nascent Am star
}

\author{
N. Przybilla ${ }^{1}$, P. Aschenbrenner ${ }^{1}$, and S. Buder ${ }^{2}$ \\ 1 Institut für Astro- und Teilchenphysik, Universität Innsbruck, Technikerstrasse 25, 6020 Innsbruck, Austria \\ e-mail: norbert .przybilla@uibk.ac . at \\ 2 Max-Planck-Institut für Astronomie, Königstuhl 17, 69117 Heidelberg, Germany
}

Received 16 June 2017 / Accepted 11 July 2017

\section{ABSTRACT}

\begin{abstract}
Direct imaging suggests that there is a Jovian exoplanet around the primary A-star in the triple-star system HD 131399. We investigate a high-quality spectrum of the primary component HD 131399A obtained with FEROS on the ESO/MPG $2.2 \mathrm{~m}$ telescope, aiming to characterise the star's atmospheric and fundamental parameters, and to determine elemental abundances at high precision and accuracy. The aim is to constrain the chemical composition of the birth cloud of the system and therefore the bulk composition of the putative planet. A hybrid non-local thermal equilibrium (non-LTE) model atmosphere technique is adopted for the quantitative spectral analysis. Comparison with the most recent stellar evolution models yields the fundamental parameters. The atmospheric and fundamental stellar parameters of HD 131399A are constrained to $T_{\text {eff }}=9200 \pm 100 \mathrm{~K}, \log g=4.37 \pm 0.10, M=1.95_{-0.06}^{+0.08} M_{\odot}$, $R=1.51_{-0.10}^{+0.13} R_{\odot}$, and $\log L / L_{\odot}=1.17 \pm 0.07$, locating the star on the zero-age main sequence. Non-LTE effects on the derived metal abundances are often smaller than $0.1 \mathrm{dex}$, but can reach up to $\sim 0.8 \mathrm{dex}$ for individual lines. The observed lighter elements up to calcium are overall consistent with present-day cosmic abundances, with a $\mathrm{C} / \mathrm{O}$ ratio of $0.45 \pm 0.07$ by number, while the heavier elements show mild overabundances. We conclude that the birth cloud of the system had a standard chemical composition, but we witness the onset of the Am phenomenon in the slowly rotating star. We furthermore show that non-LTE analyses have the potential to solve the remaining discrepancies between observed abundances and predictions by diffusion models for Am stars. Moreover, the present case allows mass loss, not turbulent mixing, to be identified as the main transport process competing with diffusion in very young Am stars.
\end{abstract}

Key words. stars: abundances - stars: atmospheres - stars: chemically peculiar - stars: early-type - stars: fundamental parameters stars: individual: HD131399

\section{Introduction}

The source HD 131399 is a member of the $16 \pm 7 \mathrm{Myr}$ old Upper Centaurus-Lupus association (UCL, de Zeeuw et al. 1999; Pecaut \& Mamajek 2016). The hierarchical triple system was only recently resolved using SPHERE on the ESO VLT by Wagner et al. (2016). It consists of the A-type primary HD 131399A and a $\sim 3.2^{\prime \prime}$ distant close pair HD 131399BC, photometrically classified as a $\mathrm{G}$ and a $\mathrm{K}$ dwarf. Moreover, the study suggested that a Jovian exoplanet, HD 131399Ab, is located at $\sim 0.8^{\prime \prime}$ distance from the primary. This is only the fourth and hottest A-type exoplanet host system detected by direct imaging (note that an alternative interpretation of HD 131399Ab as a background star has been proposed in the meantime by Nielsen et al. 2017).

Little is known about the stars of the system, with no dedicated study to be found in the literature. Estimates of atmospheric and fundamental parameters of the A-star primary were mostly published as part of photometry-based determinations of the properties of large star samples and one orbital study of the system, see Table 1 for a summary. Evidently, the scatter in the parameter values is quite large. No high-resolution spectroscopy has been obtained so far to determine elemental abundances.

The close proximity of the HD 131399BC components of $<0.1^{\prime \prime}$ complicates a quantitative study of the individual stars. To constrain the chemical composition of the birth cloud of the system, for example, as a tracer for the bulk composition of the suggested planet, the investigation therefore has to concentrate on HD 131399A. This was the original motivation for the present work, but it developed into an unexpected direction.
Table 1. Literature data for the parameters of HD 131399A.

\begin{tabular}{ll}
\hline \hline de Geus et al. (1989) & $T_{\text {eff }}=8910 \mathrm{~K}, \log g(\mathrm{cgs})=4.38$, \\
& $\log L / L_{\odot}=1.2, A_{V}=0.14 \mathrm{mag}$ \\
Kouwenhoven et al. (2007) & $M=1.82 M_{\odot}$ \\
McDonald et al. (2012) & $T_{\text {eff }}=7946 \mathrm{~K}, \log L / L_{\odot}=1.064$ \\
David \& Hillenbrand (2015) & $T_{\text {eff }}=9324 \pm 317 \mathrm{~K}, \log g=4.48$ \\
Nielsen et al. (2017) & $T_{\text {eff }}=9480_{-410}^{+420} \mathrm{~K}, \log g=4.32 \pm 0.01$, \\
& $A_{V}=0.22 \pm 0.09 \mathrm{mag}, M=2.08_{-0.11}^{+0.12} M_{\odot}$ \\
\hline
\end{tabular}

\section{Observations and data reduction}

We observed HD 131399A with the Fiber-fed Extended Range Optical Spectrograph (FEROS) on the ESO/MPG 2.2m telescope at La Silla on March 12, 2017. The measured DIMM seeing during observation was $\sim 0.6^{\prime \prime}$, resulting in no significant light from the $\sim 3.2^{\prime \prime}$ distant HD 131399BC pair falling into the $2^{\prime \prime}$ fibre aperture. Near-complete wavelength coverage from 3700 to $9200 \AA$ was achieved at resolving power $R=\lambda / \Delta \lambda=$ 48000 , with a signal-to-noise ratio $S / N \approx 250$ at $\lambda=5000 \AA$ in the $600 \mathrm{~s}$ exposure.

The basic data reduction was performed using the FEROS data reduction system (for details see Kaufer et al. 1999). The spectrum was normalised by fitting a spline function to carefully selected continuum points, taking into account that the Balmer lines are near maximum strength in HD 131399A. Finally, the spectra were shifted to the laboratory rest frame by accounting for the measured radial velocity $v_{\text {rad }}$ (see Table 2 ) as determined from cross-correlation with an appropriate synthetic spectrum. 


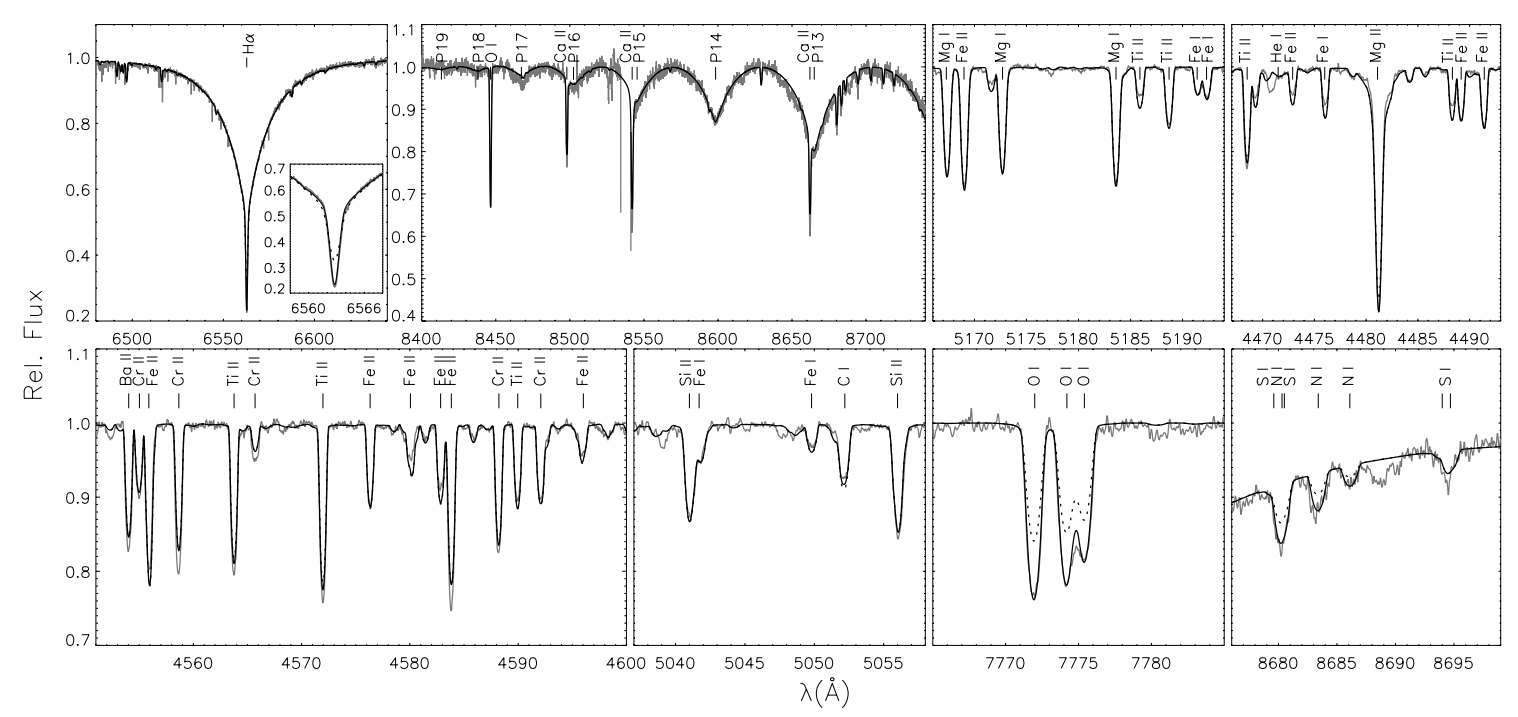

Fig. 1. Comparison of the spectrum of HD 131399 (grey) with the model computed for the final atmospheric parameters and abundances (full line) for several diagnostic regions. Pure LTE profiles (dotted) are also indicated to visualise the non-LTE effects. The inset shows the H $\alpha$ core.

\section{Model atmosphere analysis}

We employed a hybrid non-LTE approach for the model atmosphere analysis of HD 131399A as introduced to A-type stars by Przybilla et al. (2006) and used later also for the analysis of chemically peculiar early-type stars (Przybilla et al. 2008, 2016). This is based on plane-parallel homogeneous model atmospheres in hydrostatic, radiative, and local thermodynamic equilibrium (LTE) as computed with the ATLAS9 code (Kurucz 1993). In a subsequent step, non-LTE line-formation calculations were performed with recent versions of DETAIL and SURFACE (Giddings 1981; Butler \& Giddings 1985, both updated and extended by K. Butler). The coupled radiative transfer and statistical equilibrium equations were solved with DETAIL, employing the accelerated lambda iteration scheme of Rybicki \& Hummer (1991). SURFACE was then used to calculate non-LTE and LTE synthetic spectra based on refined line-broadening theories. Continuous opacities due to hydrogen and helium were considered in nonLTE, and line blocking was accounted for via LTE opacity sampling, employing the method of Kurucz (1996).

Lines for a range of important chemical species $(\mathrm{H}, \mathrm{He}$, $\mathrm{C}, \mathrm{N}, \mathrm{O}, \mathrm{Mg}, \mathrm{Si}, \mathrm{Ti}$, and $\mathrm{Fe}$ ) were treated in non-LTE (and LTE for quantifying the non-LTE-LTE abundance differences). Model atoms as discussed by Przybilla et al. (2006, 2016) were adopted. Spectral lines of 16 additional chemical elements were treated in LTE.

We employed SPAS (Spectrum Plotting and Analysing Suite, Hirsch 2009) for the comparison of synthetic spectra with observation. SPAs allows interpolating between model grid points for up to three parameters simultaneously, and instrumental and rotational broadening can be applied to the theoretical profiles. The program uses a downhill simplex algorithm to minimise $\chi^{2}$.

The atmospheric parameter determination was based on simultaneous fits to the Stark-broadened hydrogen lines and the $\mathrm{Mg}$ I/II ionisation equilibrium (requiring that the two ionisation stages indicate the same abundance) in order to constrain effective temperature $T_{\text {eff }}$ and surface gravity $\log g$ (see e.g. Przybilla et al. 2006). The microturbulence velocity $\xi$ was adjusted such that the abundances of the species treated in nonLTE became independent of the strength of the spectral lines. Line-profile fits were used for both the determination of the elemental abundances and of the projected rotational velocity $v_{\text {rot }} \sin i$. The results of the model atmosphere analysis are summarised in Table 2. Elemental abundances are averages over all analysed lines of a chemical species, giving equal weight to each spectral feature, except for manganese and iron, where only the results for the ions are considered. Uncertainties are $1 \sigma$ standard deviations from the line-to-line scatter. Individual line abundances in non-LTE and/or LTE plus more detailed information on ionic abundances can be found in the Appendix.

Based on the final atmospheric parameters and abundances, a synthetic spectrum was calculated that is compared to a selection of observed diagnostic features in Fig. 1. A similar match is achieved for practically the entire observed spectrum, the deviations reflect the line-to-line scatter of abundances. Table 2 also provides some general information on HD 131399A, such as the spectral type and the HIPPARCOS parallax, distance, and propermotion data. We note that no data on HD 131399 were provided by the first Gaia data release (Gaia Collaboration 2016) as a consequence of its multiplicity.

Starting from the photometric measurements of Slawson et al. (1992, we note that the contribution of HD 131399BC is insignificant in the optical) we furthermore constrained the interstellar reddening $E(B-V)$ and extinction $A_{V}$ (adopting a standard ratio of total-to-selective extinction of 3.1) by comparison with synthetic photometry of the model flux. From these and the known distance, we determined the absolute visual magnitude $M_{V}$ of HD 131399A, and by adopting the bolometric correction from our model atmosphere, the absolute bolometric magnitude $M_{\text {bol }}$ (see Table 2). The uncertainties of both quantities are entirely dominated by the error in the HIPPARCos parallax/distance.

In order to constrain the fundamental parameters of HD 131399A, we employed the stellar evolution models of Ekström et al. (2012). The tracks for non-rotating stars were chosen, motivated by the finding that HD 131399A is a slowly rotating star, as discussed in the next section. Figure 2 shows the location of HD 131399A in the $\log T_{\text {eff }}-\log g$ diagram, which covers the whole evolutionary sequence from the zero-age main sequence (ZAMS) to the end of core hydrogen burning. This facilitated the (evolutionary) mass $M$ to be determined. Based on this, the stellar radius $R$ and luminosity $L$ were constrained using the derived atmospheric parameters, see Table 2 . In addition, 
Table 2. Parameters and elemental abundances of HD 131399A.

\begin{tabular}{|c|c|c|c|}
\hline \multicolumn{4}{|c|}{ General information } \\
\hline \multicolumn{4}{|c|}{ Sp. Type A1 V } \\
\hline$v_{\mathrm{rad}}$ & $0.51 \pm 0.10 \mathrm{~km} \mathrm{~s}^{-1}$ & $\pi$ & $10.20 \pm 0.70$ mas $[1]$ \\
\hline$d_{\mathrm{HIP}}$ & $98.0_{-6.3}^{+7.3} \mathrm{pc}$ & $\mu_{\alpha}$ & $-29.69 \pm 0.59$ mas yr$^{-1}$ \\
\hline$d_{\text {spec }}$ & $98.0 \pm 13.0 \mathrm{pc}$ & $\mu_{\delta}$ & $-31.52 \pm 0.55$ mas yr $^{-1}[1]$ \\
\hline \multicolumn{4}{|c|}{ Atmospheric parameters } \\
\hline$T_{\text {eff }}$ & $9200 \pm 100 \mathrm{~K}$ & $\xi$ & $2 \pm 0.5 \mathrm{~km} \mathrm{~s}^{-1}$ \\
\hline $\log g(\mathrm{cgs})$ & $4.37 \pm 0.10$ & $v_{\mathrm{rot}} \sin i$ & $26 \pm 2 \mathrm{~km} \mathrm{~s}^{-1}$ \\
\hline \multicolumn{4}{|c|}{ Elemental abundances $\log (X / \mathrm{H})+12$} \\
\hline $\mathrm{He}$ & $10.99(1)$ & $\mathrm{V}^{*}$ & $4.00 \pm 0.17(2)$ \\
\hline $\mathrm{C}$ & $8.38 \pm 0.06(15)$ & $\mathrm{Cr}^{*}$ & $5.94 \pm 0.11(30)$ \\
\hline $\mathrm{N}$ & $7.65 \pm 0.05(6)$ & $\mathrm{Mn}^{*}$ & $5.88 . \pm 0.10(2)$ \\
\hline $\mathrm{O}$ & $8.73 \pm 0.04(7)$ & $\mathrm{Fe}$ & 7.70. $\pm 0.10(34)$ \\
\hline $\mathrm{Na}^{*}$ & $6.30 \pm 0.03(3)$ & $\mathrm{Co}^{*}$ & $5.32 \pm 0.03(2)$ \\
\hline $\mathrm{Mg}$ & $7.55 \pm 0.05(13)$ & $\mathrm{Ni}^{*}$ & $6.53 \pm 0.10(7)$ \\
\hline $\mathrm{Al}^{*}$ & $6.19 \pm 0.07(2)$ & $\mathrm{Cu}^{*}$ & $4.47 \pm 0.11(2)$ \\
\hline $\mathrm{Si}$ & $7.57 \pm 0.04(7)$ & $\mathrm{Zn}^{*}$ & $5.16 \pm 0.06(2)$ \\
\hline $\mathrm{S}^{*}$ & $7.22 \pm 0.14(4)$ & $\mathrm{Sr}^{*}$ & $3.18 \pm 0.03(2)$ \\
\hline $\mathrm{Ca}^{*}$ & $6.23 \pm 0.14(20)$ & $\mathrm{Y}^{*}$ & $2.14 \pm 0.23(3)$ \\
\hline $\mathrm{Sc}^{*}$ & $3.20 \pm 0.10(5)$ & $\mathrm{Zr}^{*}$ & $3.05 \pm 0.08(3)$ \\
\hline $\mathrm{Ti}$ & $5.51 \pm 0.17(29)$ & $\mathrm{Ba}^{*}$ & $3.00 \pm 0.18(5)$ \\
\hline \multicolumn{4}{|c|}{ Photometric data } \\
\hline$V$ & $7.048 \pm 0.008 \mathrm{mag}[2]$ & $A_{V}$ & $0.202 \pm 0.012 \mathrm{mag}$ \\
\hline$B-V$ & $0.118 \pm 0.007 \mathrm{mag}[2]$ & $M_{V}$ & $1.89_{-0.32}^{+0.37} \mathrm{mag}$ \\
\hline$E(B-V)$ & $0.065 \pm 0.012 \mathrm{mag}$ & $M_{\text {bol }}$ & $1.76_{-0.32}^{+0.37} \mathrm{mag}$ \\
\hline \multicolumn{4}{|c|}{ Fundamental parameters } \\
\hline$M / M_{\odot}$ & $1.95_{-0.06}^{+0.08}$ & $\log L / L_{\odot}$ & $1.17 \pm 0.07$ \\
\hline$R / R_{\odot}$ & $1.51_{-0.10}^{+0.13}$ & $\tau$ & $16 \pm 7 \mathrm{Myr}[3]$ \\
\hline
\end{tabular}

Notes. $1 \sigma$ uncertainties are given. For abundances these are from the line-to-line scatter. Numbers in brackets quantify the analysed lines. LTE abundance values are marked by an asterisk. References are indicated by square brackets.

References. (1) van Leeuwen (2007); (2) Slawson et al. (1992); (3) Pecaut \& Mamajek (2016).

the spectroscopic distance $d_{\text {spec }}$ was derived employing Eq. (1) of Nieva \& Przybilla (2012). Its uncertainty is dominated by the error bars of $\log g$. The spectroscopic distance is in excellent agreement with the independent HIPPARCOS measurement, thus ensuring that a consistent solution for the atmospheric and fundamental parameters was found.

Because of the position of HD 131399A on the ZAMS - in agreement with expectation - we cannot employ an isochrone fit to determine its age $\tau$. Instead, the independent age estimate for the UCL association by Pecaut \& Mamajek (2016) was adopted, which accounts for the intrinsic age spread of the members of UCL. From this and the stellar evolution models, we find that HD 131399A has spent only $\sim 1 \%$ of its lifetime so far.

\section{Discussion}

Our values from high-resolution spectroscopy are compatible with previous photometry-based atmospheric parameter estimates (Fig. 2), but with significantly reduced uncertainties in $T_{\text {eff }}$. Our observations facilitate a determination of $v_{\text {rot }} \sin i$ for the first time. Even though Wagner et al. (2016) provided only loose constraints on the inclination angle from their orbital characterization of the system, $i=45^{\circ}$ to $65^{\circ}$ for the orbit of HD 131399BC around HD 131399A, this is sufficient to place an upper limit of $v_{\text {rot }}<40 \mathrm{~km} \mathrm{~s}^{-1}$ assuming a co-alignment of the

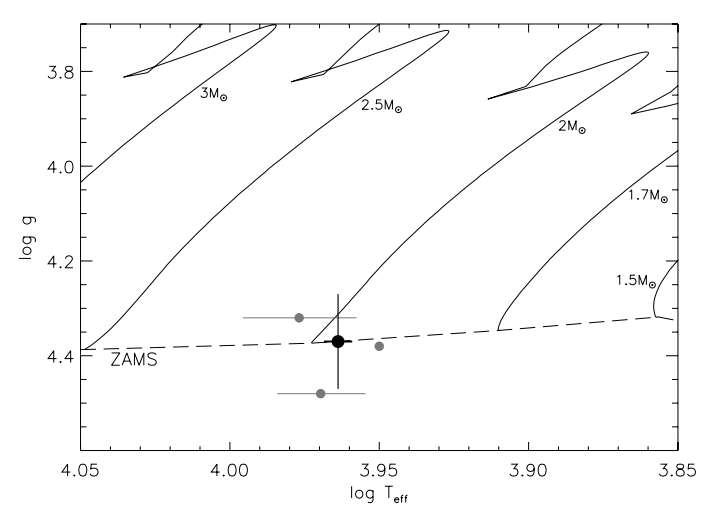

Fig. 2. HD 131399A in the $\log T_{\text {eff }}-\log g$ diagram (black dot). Overlaid are Geneva evolution tracks for non-rotating stars, computed for metallicity $Z=0.014$ (Ekström et al. 2012, full lines), spanning the width of the main sequence. The dashed line marks the zero-age main sequence. Results from previous parameter determinations (Table 1) are marked by grey dots. Where available, $1 \sigma$ error bars are displayed.

star's rotational axis with the axis of the orbital motion. This means that HD 131399A is a slowly rotating star.

We present the most complete non-LTE abundance determination in an A star to date. A third of the observed chemical species and about half of the total spectral lines was treated in non-LTE, thus improving the accuracy and precision of the resulting abundance data.

As a very young and nearby star, we would expect HD 131399 to show pristine abundances such as those typical today in the solar neighbourhood (Nieva \& Przybilla 2012; Przybilla et al. 2013). Figure 3 shows a comparison of the derived abundances with this cosmic abundance standard (CAS), supplemented by meteoritic solar abundances (Asplund et al. 2009) where data from young massive stars are unavailable. The expected range of abundance values is highlighted by the grey band, with some deviations to be considered for elements where only LTE values are available. The lighter elements up to $\mathrm{Ca}$ are overall compatible with expectation. In particular, the $\mathrm{C} / \mathrm{O}$ ratio is found to be $0.45 \pm 0.07$ by number, in good agreement with the CAS average of $0.43 \pm 0.06$. However, marked overabundances become apparent for the heavier elements, up to a factor of $\sim 6$ for Ba. Enrichment levels for the lanthanides are apparently not high enough to produce detectable lines.

As an early A-type star, HD 131399A has a very thin surface convection zone that is prone to develop chemical peculiarities for $v_{\text {rot }} \lesssim 120 \mathrm{~km} \mathrm{~s}^{-1}$ (Abt 2000). This can be a fast process occurring on timescales as short as some $10^{6}$ years, that is, even in the pre-main-sequence-phase (Vick et al. 2011). The reason is that atomic diffusion (e.g. Michaud 1970) is apparently important in HD 131399A even though it has avoided classification as an Am star. HD 131399A does not show the abundance pattern characteristic for Am stars - marked underabundances for $\mathrm{C}$ and $\mathrm{Sc}$, underabundances for $\mathrm{O}$ and $\mathrm{Ca}$, and overabundances of the iron-group elements - as discussed by Vick et al. (2010, and references therein), for instance. However, typical Am stars have ages of the order of several $10^{8} \mathrm{yr}$, while the spectral characteristics are still developing in HD 131399A.

Stellar models accounting for atomic diffusion may provide some guidance for the interpretation. Figure 15 of Richer et al. (2000) and Fig. 14 of Vick et al. (2010), for example, discuss the evolution of the surface abundances as a function of age. While these models are not tailored to the parameters of HD 131399A, they should be close enough to describe the effects qualitatively. The models of Richer et al. and Vick et al. in the age range 


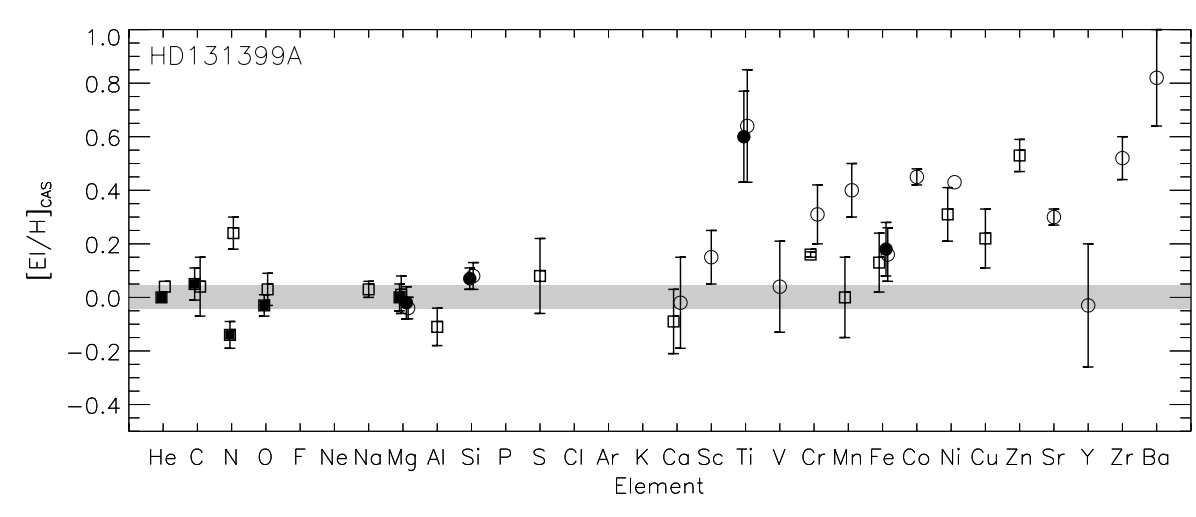

Fig. 3. Elemental abundances in HD 131399A, relative to the cosmic abundance standard (CAS Nieva \& Przybilla 2012; Przybilla et al. 2013), supplemented by solar meteoritic abundances (Asplund et al. 2009) where unavailable from young massive stars. The grey bar spans the expected CAS range. Non-LTE abundances for HD 131399A are indicated by filled, and LTE values by open symbols. Squares mark data based on neutral species, and circles data from single-ionized species. Error bars are $1 \sigma$ uncertainties as deduced from from the line-to-line scatter. of HD 131399A indeed show the observed pattern, nearly pristine abundances up to calcium, and mild overabundances of the iron-group elements. Models with either turbulent mixing or mass loss as the main competitor to atomic diffusion make very similar predictions for Am stars at later ages (Michaud et al. 2011), but there are some distinct features at early times. In particular, a large overabundance of titanium is unique (Vick et al. 2010). We can therefore identify mass loss as the main transport process counter-acting diffusion in very young Am stars. We conclude that HD 131399A can be viewed as a rare but very valuable case of a nascent Am star. The present work provides tight observational constraints for atomic diffusion in very young stars that start to develop the Am phenomenon. We would like to suggest further investigations of the fast development of the titanium abundance in diffusion models, as this could provide an accurate and precise age indicator for nascent Am stars, a "titanium clock", and a means to constrain otherwise unaccessible mass-loss rates in young A-type stars.

Concerning our initial motivation for this study, we may conclude that it is probable that the pristine abundances for the heavier elements of the iron group and beyond were also compatible with CAS/solar abundances in view of the ongoing diffusion processes. The birth cloud out of which the entire system HD 131399 including the candidate planet formed most likely showed a standard composition as typical for the present-day solar neighbourhood.

Finally, we would like to discuss the non-LTE effects on the abundance analysis, as these were studied to such large extent in an Am star for the first time. This is visualised in Fig. 1, where the differences between non-LTE and LTE model spectra are shown, in Fig. 3, where the ionic abundances in non-LTE and LTE are compared, and in Table A.1, where the details from the line-by-line analysis are summarised. Non-LTE effects on average abundances are usually small in HD 131399 for most of the elements. They amount to $\lesssim 0.1 \mathrm{dex}$, if some spectral lines with obviously discrepant LTE abundance values are not considered. These outliers include classical features such as the O I $\lambda \lambda 7771-5 \AA$ triplet and O I $\lambda 8446 \AA$, which show a pronounced non-LTE-LTE abundance difference $\Delta \log \varepsilon$ by $\sim 0.8$ dex (for a detailed discussion see e.g. Przybilla et al. 2000). Other (less extreme) examples are the red Si II $\lambda \lambda 6347,6371 \AA$ lines, which show $\Delta \log \varepsilon \approx 0.25$ dex. However, for N I, all lines are affected almost uniformly by rather large non-LTE effects, $\Delta \log \varepsilon \approx 0.4 \mathrm{dex}$ (see e.g. Przybilla \& Butler 2001). This has the potential to solve one of the most prominent discrepancies between observation and predictions from diffusion models for Am stars (Richer et al. 2000; Vick et al. 2010, and references therein). Discrepancies in the ionisation equilibria of some elements that can be currently analysed in LTE only hint on the presence of (substantial) non-LTE effects for these as well.
We have to state, however, that the differences between nonLTE and LTE abundances found here should not be generalised throughout the Am star parameter space, but trends can be estimated. Non-LTE effects might well become stronger for the typically (much) more evolved Am stars. On the one hand, the lower-density plasma at lower gravities supports less thermalising collisions, but on the other hand, the overall higher atmospheric abundances for most elements will extend the lineformation region outwards in the atmospheres, where non-LTE departures are more pronounced. We conclude that additional non-LTE studies of Am stars (also covering more elements) will be worthwhile to provide unbiased abundances for the comparison with diffusion models, which may in turn open up possibilities for refining the models as well.

Acknowledgements. S.B. acknowledges funding from the Alexander von Humboldt Foundation in the framework of a Sofja Kovalevskaja Award endowed by the German Federal Ministry of Education and Research.

\section{References}

Abt, H. A. 2000, ApJ, 544, 933

Asplund, M., Grevesse, N., Sauval, A. J., \& Scott, P. 2009, ARA\&A, 47, 481 Bates, D., \& Damgaard, A. 1949, Phil. Trans. Roy. Soc., 242A, 101 Biemont, E., Grevesse, N., Hannaford, P., \& Lowe, R. M. 1981, ApJ, 248, 867 Butler, K., \& Giddings, J. R. 1985, in Newsletter of Analysis of Astronomical Spectra, No. 9 (Univ. London)

David, T. J., \& Hillenbrand, L. A. 2015, ApJ, 804, 146

Davidson, M. D., Snoek, L. C., Volten, H., \& Doenszelmann, A. et al. 1992, A\&A, 255, 457

de Geus, E. J., de Zeeuw, P. T., \& Lub, J. 1989, A\&A, 216, 44

de Zeeuw, P. T., Hoogerwerf, R., de Bruijne, J. H. J., et al. 1999, AJ, 117, 354

Ekström, S., Georgy, C., Eggenberger, P., et al. 2012, A\&A, 537, A146

Froese Fischer, C., \& Tachiev, G. 2004, At. Data Nucl. Data Tables, 87, 1

Froese Fischer, C., Tachiev, G., \& Irimia, A. 2006, At. Data Nucl. Data Tables, 92, 607

Fuhr, J. R., Martin, G. A., \& Wiese, W. L. 1988, J. Phys. Chem. Ref. Data, 17, Suppl. 4

Fuhr, J. R., \& Wiese, W. L. 1998, in CRC Handbook of Chemistry and Physics, 79th edn., ed. D. R. Lide (Boca Raton: CRC Press)

Gaia Collaboration (Brown, A. G. A., et al.) 2016, A\&A, 595, A2

Giddings, J. R. 1981, Ph.D. Thesis, Univ. London

Hannaford, P., Lowe, R. M., Grevesse, N., Biemont, E., \& Whaling, W. 1982, ApJ, 261, 736

Hirsch, H. 2009, Ph.D. Thesis, Univ. Erlangen-Nuremberg

Kaufer, A., Stahl, O., Tubbesing, S., et al. 1999, The Messenger, 95, 8

Kerkhoff, H., Schmidt, M., \& Zimmermann, P. 1980, Z. Phys. A, 298, 249

Kramida, A., Ralchenko, Yu., Reader, J., \& NIST ASD Team 2016, NIST Atomic Spectra Database (v5.4), http://physics.nist.gov/asd (NIST, Gaithersburg, MD)

Kouwenhoven, M. B. N., Brown, A. G. A., Portegies Zwart, S. F. \& Kapel, L. 2007, A\&A, 474, 77

Kurucz, R. L. 1993, CD-ROM No. 13 (Cambridge, Mass.: SAO)

Kurucz, R. L., \& Bell, B. 1995, CD-ROM No. 23 (Cambridge, Mass: SAO)

Kurucz, R. L. 1996, ASP Conf. Ser., 108, 160 
N. Przybilla et al.: Candidate exoplanet host HD 131399A: a nascent Am star

Luo, D., \& Pradhan, A. K. 1989, J. Phys. B, 22, 3377

Martin, G. A., Fuhr, J. R., \& Wiese, W. L. 1988, J. Phys. Chem. Ref. Data, 17, Suppl. 3

McDonald, I., Zijlstra, A. A., \& Boyer, M. L. 2012, MNRAS, 427, 343

Michaud, G. 1970, ApJ, 160, 641

Michaud, G., Richer, J., \& Vick, M. 2011, A\&A, 534, A18

Nielsen, E. L., De Rosa, R. J., Rameau, J., et al. 2017, AJ, submitted [arXiv: 1705.06851]

Nieva, M. F., \& Przybilla, N. 2012, A\&A, 539, A143

Pecaut, M. J., \& Mamajek, E. E. 2016, MNRAS, 461, 794

Pickering, J. C., Thorne, A. P., \& Perez, R. 2001, ApJS, 132, 403

Pickering, J. C., Thorne, A. P., \& Perez, R. 2002, ApJS, 138, 247

Przybilla, N., \& Butler, K. 2001, A\&A, 379, 955

Przybilla, N., Butler, K., Becker, S. R., et al. 2000, A\&A, 359, 1085

Przybilla, N., Butler, K., Becker, S. R., \& Kudritzki, R. P. 2006, A\&A, 445, 1099
Przybilla, N., Nieva, M. F., Tillich, A., et al. 2008, A\&A, 488, L51

Przybilla, N., Nieva, M. F., Irrgang, A., \& Butler, K. 2013, EAS PS, 63, 13

Przybilla, N., Fossati, L., Hubrig, S., et al. 2016, A\&A, 587, A7

Richer, J., Michaud, G., \& Turcotte, S. 2000, ApJ, 529, 338

Rybicki, G. B., \& Hummer, D. G. 1991, A\&A, 245, 171

Slawson, R. W., Hill, R. J., \& Landstreet, J. D. 1992, ApJS, 82, 117

van Leeuwen, F. 2007, A\&A, 474, 653

Vick, M., Michaud, G., Richer, J., \& Richard, O. 2010, A\&A, 521, A62

Vick, M., Michaud, G., Richer, J., \& Richard, O. 2011, A\&A, 526, A37

Wiese, W. L., \& Fuhr, J. R. 2009, J. Phys. Chem. Ref. Data, 38, 565

Wiese, W. L., Smith, M. W., \& Miles, B. M. 1969, Atomic transition probabilities, Vol. 2, Nat. Stand. Ref. Data Ser., Nat. Bur. Stand. (US), NSRDS-NBS 22

Wiese, W. L., Fuhr, J. R., \& Deters, T. M. 1996, J. Phys. Chem. Ref. Data, Mon., 7

Wagner, K., Apai, D., Kasper, M., et al. 2016, Science, 353, 673 


\section{Appendix A: Line-by-line analysis}

Table A.1 summarises the results from the abundance analysis of individual lines of the different chemical species observed in HD 131399A. For each ion the transition wavelengths $\lambda$ are given, together with the excitation potential of the lower level $\chi$, the adopted oscillator strength $\log g f$ for the transition, an accuracy indicator and the reference to the source of the oscillator strength, the abundance $\log \varepsilon=\log (X / \mathrm{H})+12$ (in non-LTE, if a model atom is available, otherwise in LTE), and the difference between non-LTE and LTE abundance, $\Delta \log \varepsilon,=\log \varepsilon_{\text {NLTE }}-$ $\log \varepsilon_{\text {LTE }}$. When several fine-structure transitions contribute to an observed line, abundance data are given at the first entry only. For each ion, the total abundance is also indicated in non-LTE and/or LTE, averaged using the same weight for all lines, and the $1 \sigma$ uncertainty from the line-to-line scatter. The number of analysed lines is given in brackets. Some lines show obvious nonLTE effects when compared to the other transitions in an ion. In these cases, the line is marked in italics and is discarded from the LTE average, as would be done in a classical LTE study.

Table A.1. Line-by-line elemental abundances.

\begin{tabular}{|c|c|c|c|c|c|c|}
\hline$\lambda(\AA)$ & $\chi(\mathrm{eV})$ & $\log g f$ & Acc. & Ref. & $\log \varepsilon$ & $\Delta \log \varepsilon$ \\
\hline He I: & \multicolumn{4}{|c|}{$\log \varepsilon_{\mathrm{NLTE}}=10.99(1)$} & \multicolumn{2}{|c|}{$\log \varepsilon_{\mathrm{LTE}}=11.03(1)$} \\
\hline 5875.599 & 20.96 & -1.516 & AAA & WF & 10.99 & -0.04 \\
\hline 5875.614 & 20.96 & -0.340 & AAA & WF & & \\
\hline 5875.615 & 20.96 & 0.409 & AAA & WF & & \\
\hline 5875.625 & 20.96 & -0.339 & AAA & WF & & \\
\hline 5875.640 & 20.96 & 0.138 & AAA & WF & & \\
\hline 5875.966 & 20.96 & -0.214 & AAA & WF & & \\
\hline \multicolumn{4}{|c|}{$\mathrm{C} \mathrm{I}: \log \varepsilon_{\mathrm{NLTE}}=8.38 \pm 0.06(15)$} & \multicolumn{3}{|c|}{$\log \varepsilon_{\mathrm{LTE}}=8.37 \pm 0.11(15)$} \\
\hline 4771.742 & 7.49 & -1.866 & $\mathrm{C}$ & WFD & 8.45 & 0.02 \\
\hline 4775.898 & 7.49 & -2.304 & $\mathrm{C}$ & WFD & 8.3 & 0.02 \\
\hline 4932.049 & 7.68 & -1.658 & B & WFD & & 0.06 \\
\hline 5052.16 & 7.68 & -1.303 & B & WFD & & 0.07 \\
\hline 5668.951 & 8.54 & -1.429 & $\mathrm{C}$ & LP & 8.3 & 0.03 \\
\hline 6013.166 & 8.65 & -1.314 & $\mathrm{D}$ & WFD & 8.37 & 0.05 \\
\hline 6013.213 & 8.65 & -1.673 & $\mathrm{D}$ & WFD & & \\
\hline 6014.830 & 8.64 & -1.585 & D & WFD & & 0.02 \\
\hline 6587.610 & 8.54 & -1.003 & B & WFD & 8. & 0.09 \\
\hline 7100.124 & 8.64 & -1.470 & B & WFD & 8.47 & 0.03 \\
\hline 7108.934 & 8.64 & -1.592 & $\mathrm{~B}$ & WFD & 8.3 & 0.02 \\
\hline 7111.472 & 8.64 & -1.086 & $\mathrm{~B}-$ & WFD & 8.4 & 0.02 \\
\hline 7113.178 & 8.65 & -0.774 & $\mathrm{~B}-$ & WFD & 8.2 & 0.05 \\
\hline 9078.288 & 7.48 & -0.581 & $\mathrm{~B}$ & WFD & 8.40 & -0.09 \\
\hline 9088.513 & 7.48 & -0.429 & B & WFD & 8.39 & -0.11 \\
\hline 9111.807 & 7.49 & -0.298 & $\mathrm{~B}$ & WFD & 8.44 & -0.14 \\
\hline
\end{tabular}

Notes. Accuracy indicators - uncertainties within AAA: 0.3\%; AA: $1 \%$; A: 3\%; B: 10\%; C: 25\%; D: 50\%; E: larger than 50\%; X: unknown.

References. B: Biemont et al. (1981); CA: Coulomb approximation, Bates \& Damgaard (1949); D: Davidson et al. (1992); FFT: Froese Fischer \& Tachiev (2004); FFTI: Froese Fischer et al. (2006); FMW: Fuhr et al. (1988); FW: Fuhr \& Wiese (1998); H: Hannaford et al. (1982); KB: Kurucz \& Bell (1995); KSZ: Kerkhoff et al. (1980); LP: Luo \& Pradhan (1989); MFW: Martin et al. (1988); NIST: Kramida et al. (2016); OP: Opacity Project, available electronically via TOPBASE (http://cdsweb.u-strasbg.fr/topbase/ topbase.html); P: Pickering et al. (2001, 2002); WF: Wiese \& Fuhr (2009); WFD: Wiese et al. (1996); WSM: Wiese et al. (1969).
Table A.1. continued.

\begin{tabular}{|c|c|c|c|c|c|c|}
\hline$\lambda(\AA)$ & $\chi(\mathrm{eV})$ & $\log g f$ & Acc. & Ref. & $\log \varepsilon$ & $\Delta \log \varepsilon$ \\
\hline \multicolumn{7}{|c|}{ N I: $\quad \log \varepsilon_{\text {NLTE }}=7.65 \pm 0.05(6)$} \\
\hline 7442.298 & 10.33 & -0.384 & $\mathrm{~B}+$ & WFD & 7.59 & -0.45 \\
\hline 8594.000 & 10.68 & -0.334 & $\mathrm{~B}$ & WFD & 7.59 & -0.38 \\
\hline 8629.235 & 10.69 & 0.075 & $\mathrm{~B}$ & WFD & 7.61 & -0.42 \\
\hline 8683.403 & 10.33 & 0.086 & $\mathrm{~B}+$ & WFD & 7.70 & -0.44 \\
\hline 8686.149 & 10.33 & -0.305 & $\mathrm{~B}+$ & WFD & 7.63 & -0.35 \\
\hline 8703.247 & 10.33 & -0.322 & $\mathrm{~B}+$ & WFD & 7.69 & -0.34 \\
\hline \multicolumn{7}{|c|}{ O I: $\quad \log \varepsilon_{\mathrm{NLTE}}=8.73 \pm 0.04(7), \quad \log \varepsilon_{\mathrm{LTE}}=8.79 \pm 0.06(4)$} \\
\hline 6155.961 & 10.74 & -1.363 & $\mathrm{~B}+$ & WFD & 8.67 & -0.05 \\
\hline 6155.971 & 10.74 & -1.011 & $\mathrm{~B}+$ & WFD & & \\
\hline 6155.989 & 10.74 & -1.120 & $\mathrm{~B}+$ & WFD & & \\
\hline 6156.737 & 10.74 & -1.487 & $\mathrm{~B}+$ & WFD & & \\
\hline 6156.755 & 10.74 & -0.898 & $\mathrm{~B}+$ & WFD & & \\
\hline 6156.788 & 10.74 & -0.694 & $\mathrm{~B}+$ & WFD & & \\
\hline 6158.149 & 10.74 & -1.841 & $\mathrm{~B}+$ & WFD & 8.72 & -0.06 \\
\hline 6158.172 & 10.74 & -0.995 & $\mathrm{~B}+$ & WFD & & \\
\hline 6158.187 & 10.74 & -0.409 & $\mathrm{~B}+$ & WFD & & \\
\hline 6453.602 & 10.74 & -1.288 & $\mathrm{C}+$ & WFD & 8.80 & -0.07 \\
\hline 6454.444 & 10.74 & -1.066 & $\mathrm{C}+$ & WFD & & \\
\hline 7001.899 & 10.99 & -1.489 & B & WFD & 8.74 & -0.03 \\
\hline 7001.922 & 10.99 & -1.011 & B & WFD & & \\
\hline 7002.173 & 10.99 & -2.664 & B & WFD & & \\
\hline 7002.196 & 10.99 & -1.489 & B & WFD & & \\
\hline 7002.230 & 10.99 & -0.741 & B & WFD & & \\
\hline 7002.250 & 10.99 & -1.364 & B & WFD & & \\
\hline 7771.944 & 9.15 & 0.354 & A & FFT & 8.71 & -0.75 \\
\hline 7774.166 & 9.15 & 0.207 & A & FFT & 8.75 & -0.71 \\
\hline 7775.388 & 9.15 & -0.015 & A & FFT & & \\
\hline 8446.247 & 9.52 & -0.468 & B & FFT & 8.69 & -0.83 \\
\hline 8446.359 & 9.52 & 0.231 & B & FFT & & \\
\hline 8446.758 & 9.52 & 0.009 & B & FFT & & \\
\hline \multicolumn{4}{|l|}{ Na I: } & \multicolumn{3}{|c|}{$\log \varepsilon_{\mathrm{LTE}}=6.30 \pm 0.03(3)$} \\
\hline 5688.193 & 2.10 & -1.407 & & FFTI & 6.28 & \\
\hline 5688.204 & 2.10 & -0.453 & A & FFTI & & \\
\hline 5889.951 & 0.00 & 0.108 & AA & NIST & 6.84 & \\
\hline 5895.924 & 0.00 & -0.194 & AA & NIST & 6.75 & \\
\hline 8183.256 & 2.10 & -0.235 & $\mathrm{~A}+$ & FFTI & 6.30 & \\
\hline 8194.790 & 2.10 & -0.464 & A & FFTI & 6.33 & \\
\hline 8194.824 & 2.10 & 0.491 & $\mathrm{~A}+$ & FFTI & & \\
\hline \multicolumn{7}{|c|}{$\mathrm{Mg}$ I: $\log \varepsilon_{\mathrm{NLTE}}=7.56 \pm 0.05(5)$} \\
\hline 4702.991 & 4.35 & -0.666 & $\mathrm{X}$ & KB & 7.58 & 0.01 \\
\hline 5172.684 & 2.71 & -0.393 & $\mathrm{~B}+$ & NIST & 7.61 & -0.03 \\
\hline 5183.604 & 2.72 & -0.167 & A & NIST & 7.55 & -0.05 \\
\hline 5528.405 & 4.35 & -0.620 & $\mathrm{X}$ & $\mathrm{KB}$ & 7.48 & 0.03 \\
\hline 8806.766 & 4.35 & -0.134 & A & NIST & 7.59 & 0.00 \\
\hline \multicolumn{7}{|c|}{$\mathrm{Mg}$ II $: \log \varepsilon_{\mathrm{NLTE}}=7.54 \pm 0.06(8), \quad \log \varepsilon$} \\
\hline 3848.211 & 8.86 & -1.495 & $\mathrm{C}$ & OP & 7.47 & 0.00 \\
\hline 3848.340 & 8.86 & -2.398 & $\mathrm{D}$ & OP & & \\
\hline 4390.514 & 10.00 & -1.70 & $\mathrm{D}$ & WSM & 7.50 & 0.04 \\
\hline 4390.572 & 10.00 & -0.530 & A & FFTI & & \\
\hline 4427.994 & 10.00 & -1.208 & A & FFTI & 7.53 & 0.04 \\
\hline 4433.988 & 10.00 & -0.907 & A & FFTI & 7.54 & 0.04 \\
\hline 4481.126 & 8.86 & 0.730 & B & FW & 7.51 & -0.04 \\
\hline 4481.150 & 8.86 & -0.570 & B & FW & & \\
\hline 4481.325 & 8.86 & 0.575 & B & FW & & \\
\hline 6545.968 & 11.63 & 0.41 & $\mathrm{C}$ & $\mathrm{CA}$ & 7.65 & 0.11 \\
\hline
\end{tabular}


Table A.1. continued.

Table A.1. continued.

\begin{tabular}{|c|c|c|c|c|c|c|c|c|c|c|c|c|c|}
\hline$\lambda(\AA)$ & $\chi(\mathrm{eV})$ & $\log g f$ & Acc. & Ref. & $\log \varepsilon$ & $\Delta \log \varepsilon$ & $\lambda(\AA)$ & $\chi(\mathrm{eV})$ & $\log g f$ & Acc. & Ref. & $\log \varepsilon$ & $\Delta \log \varepsilon$ \\
\hline 7877.054 & 10.00 & 0.391 & $A+$ & FFTI & 7.54 & -0.01 & \multicolumn{4}{|c|}{ Ti II: $\log \varepsilon_{\mathrm{NLTE}}=5.51 \pm 0.17(29)$} & \multicolumn{3}{|c|}{$\log \varepsilon_{\mathrm{LTE}}=5.55 \pm 0.21(29)$} \\
\hline 7896.040 & 10.00 & -0.308 & A & FFTI & 7.58 & 0.01 & 3900.539 & 1.13 & -0.20 & $\mathrm{~B}$ & $\mathrm{P}$ & 5.63 & -0.17 \\
\hline 7896.366 & 10.00 & 0.647 & $\mathrm{~A}+$ & FFTI & & & 4028.343 & 1.89 & -1.00 & $\mathrm{D}$ & MFW & 5.39 & 0.00 \\
\hline Al I: & & & & $\log \varepsilon_{\mathrm{LTE}}$ & $=6.19 \pm$ & $0.07(2)$ & 4163.644 & 2.59 & -0.13 & $\mathrm{~B}$ & $\mathrm{P}$ & 5.43 & -0.18 \\
\hline 3944.006 & 0.00 & -0.64 & $\mathrm{C}+$ & WSM & 6.24 & & 4171.904 & 2.60 & -0.29 & B & $\mathrm{P}$ & 5.60 & -0.08 \\
\hline 3961.520 & 0.01 & -0.337 & $\mathrm{C}+$ & WSM & 6.14 & & 4287.872 & 1.08 & -2.02 & D- & MFW & 5.54 & 0.02 \\
\hline \multirow{2}{*}{\multicolumn{4}{|c|}{ Si II: $\quad \log \varepsilon_{\text {NLTE }}=7.57 \pm 0.04(7)$}} & \multirow{2}{*}{\multicolumn{3}{|c|}{$\log \varepsilon_{\mathrm{LTE}}=7.58 \pm 0.05(5)$}} & 4294.099 & 1.08 & -1.11 & D- & MFW & 5.86 & -0.10 \\
\hline & & & & & & & 4300.042 & 1.18 & -0.44 & B & $\mathrm{P}$ & 5.67 & -0.07 \\
\hline 3853.665 & 6.86 & -1.397 & $\mathrm{C}$ & FFTI & 7.52 & -0.03 & 4301.922 & $\begin{array}{l}1.10 \\
1.16\end{array}$ & -1.15 & $\mathrm{~B}$ & $\mathrm{P}$ & 5.43 & -0.03 \\
\hline 3862.595 & 6.86 & -0.757 & $\mathrm{C}+$ & NIST & 7.59 & -0.06 & 4312.856 & 1.18 & -1.10 & B & $\mathrm{P}$ & 5.51 & -0.05 \\
\hline 5055.984 & 10.07 & 0.42 & $\mathrm{D}+$ & WSM & 7.62 & 0.01 & 4395.033 & 1.08 & -0.66 & D- & MFW & 5.80 & -0.09 \\
\hline 5056.317 & 10.07 & -0.53 & $\mathrm{E}$ & WSM & & & 4399.772 & 1.24 & -1.27 & D- & MFW & 5.39 & -0.01 \\
\hline 5957.559 & 10.07 & -0.36 & $\mathrm{D}$ & WSM & 7.59 & 0.02 & 4443.794 & 1.08 & -0.70 & D- & MFW & 5.51 & -0.12 \\
\hline 5978.930 & 10.07 & -0.06 & $\mathrm{D}$ & WSM & 7.56 & 0.03 & $\begin{array}{l}4445.194 \\
4450.482\end{array}$ & $\begin{array}{l}1.00 \\
1.08\end{array}$ & -1.52 & $\mathrm{~B}$ & $\mathrm{P}$ & 5.38 & 0.00 \\
\hline 6347.109 & 8.12 & 0.176 & $\mathrm{C}+$ & FFTI & 7.62 & -0.25 & 4468.507 & 1.13 & -0.60 & D- & MFW & 5.57 & -0.11 \\
\hline 6371.371 & 8.12 & -0.126 & $\mathrm{C}+$ & FFTI & 7.53 & -0.23 & 4488.331 & 3.11 & -0.82 & D- & MFW & 5.52 & 0.00 \\
\hline S I: & & & & \multicolumn{3}{|c|}{$\log \varepsilon_{\mathrm{LTE}}=7.22 \pm 0.14$} & 4501.273 & 1.12 & -0.75 & D- & MFW & 5.61 & -0.09 \\
\hline 6052.66 & 7.87 & -0.393 & $X$ & FW & 7.12 & $\ldots$ & 4529.474 & 1.57 & -2.03 & D- & MFW & 5.67 & 0.02 \\
\hline 6748.58 & 7.87 & -0.803 & $X$ & FW & 7.21 & $\ldots$ & 4563.761 & 1.22 & -0.96 & D- & MFW & 5.76 & 0.03 \\
\hline 6748.79 & 7.87 & -0.596 & $\mathrm{X}$ & FW & & & 4571.971 & 1.57 & -0.32 & B & $\mathrm{P}$ & 5.81 & -0.12 \\
\hline 6756.75 & 7.87 & -1.745 & $\mathrm{X}$ & $\mathrm{FW}$ & 7.13 & & 4589.958 & 1.24 & -1.79 & D- & MFW & 5.47 & 0.00 \\
\hline 6756.96 & 7.87 & -0.905 & $\mathrm{X}$ & $\mathrm{FW}$ & & & 4779.985 & 2.05 & -1.37 & D- & MFW & 5.33 & 0.00 \\
\hline 6757.15 & 7.87 & -0.307 & $X$ & $\mathrm{FW}$ & & & 4798.532 & 1.08 & -2.68 & $\mathrm{C}$ & $\mathrm{P}$ & 5.35 & 0.02 \\
\hline 8693.16 & 7.87 & -1.377 & $X$ & $\mathrm{FW}$ & 7.42 & $\ldots$ & 4805.085 & 2.06 & -1.10 & D- & MFW & 5.43 & -0.03 \\
\hline 8693.98 & 7.87 & -0.521 & $\mathrm{X}$ & FW & & & 4911.193 & 3.12 & -0.61 & B & $\mathrm{P}$ & 5.20 & 0.02 \\
\hline 8694.71 & 7.87 & 0.050 & $X$ & FW & & & 5010.211 & 3.09 & -1.29 & $\mathrm{C}$ & $\mathrm{P}$ & 5.27 & 0.01 \\
\hline Ca I: & & & & $\log \varepsilon_{\mathrm{LTE}}=$ & $620+$ & $.12(11)$ & 5129.152 & 1.89 & -1.39 & D- & MFW & 5.45 & 0.01 \\
\hline 4226.728 & 0.00 & 0.244 & $\mathrm{~B}+$ & NIST & 6.48 & & 5185.913 & 1.89 & -1.35 & $\mathrm{D}$ & MFW & 5.24 & 0.00 \\
\hline 4318.652 & 1.90 & -0.21 & $\mathrm{C}+$ & NIST & $\begin{array}{l}0.40 \\
6.23\end{array}$ & & 5188.680 & 1.58 & -1.21 & D- & MFW & 5.55 & -0.06 \\
\hline 4425.437 & 1.88 & -0.358 & $\mathrm{C}$ & FW & 6.25 & & 5336.771 & 1.58 & -1.70 & D- & MFW & 5.31 & 0.01 \\
\hline 4434.957 & 1.88 & -0.01 & $\mathrm{C}$ & FW & 6.15 & $\cdots$ & V II: & & & & \multicolumn{3}{|c|}{$\log \varepsilon_{\mathrm{LTE}}=4.00 \pm 0.17(2)$} \\
\hline 4581.395 & 2.52 & -0.337 & $\mathrm{C}$ & NIST & 6.10 & -5 & 4023.388 & 1.80 & -0.52 & $\mathrm{X}$ & $\mathrm{KB}$ & 3.88 & $\ldots$ \\
\hline 4581.467 & 2.52 & -1.26 & $\mathrm{D}$ & NIST & & & 4183.440 & 2.05 & -0.95 & $X$ & $\mathrm{~KB}$ & 4.12 & \\
\hline 4585.865 & 2.53 & -0.187 & $\mathrm{C}$ & NIST & 6.00 & & Cr I: & & & & \multicolumn{3}{|c|}{$\log \varepsilon_{\mathrm{LTE}}=5.80 \pm 0.01$} \\
\hline 4585.964 & 2.53 & -1.26 & $\mathrm{D}$ & NIST & & & 4254.331 & 0.00 & -0.11 & $\mathrm{~B}$ & MFW & 5.81 & $\ldots$ \\
\hline 4586.036 & 2.53 & -2.81 & $\mathrm{E}$ & NIST & & & 4274.806 & 0.00 & -0.23 & $\mathrm{~B}$ & MFW & 5.79 & $\ldots$ \\
\hline 5857.451 & 2.93 & 0.23 & $\mathrm{D}$ & NIST & 6.27 & $\ldots$ & 5204.505 & 0.94 & -0.20 & B & MFW & 5.80 & $\ldots$ \\
\hline 6122.217 & 1.89 & -0.315 & $\mathrm{C}$ & FW & 6.18 & $\cdots$ & Cr II: & & & & \multicolumn{3}{|c|}{$\log \varepsilon_{\mathrm{LTE}}=5.95 \pm 0.11(27)$} \\
\hline 6162.173 & 1.90 & -0.089 & C & FW & 6.23 & $\cdots$ & 4037.972 & 6.49 & -0.557 & $X$ & KB & 5.75 & . \\
\hline 6439.075 & 2.53 & 0.47 & D & NIST & 6.19 & $\cdots$ & 4051.930 & 3.09 & -2.192 & $\mathrm{X}$ & $\mathrm{KB}$ & 5.77 & $\cdots$ \\
\hline 6462.567 & 2.52 & 0.31 & $\mathrm{D}$ & NIST & 6.15 & & 4242.364 & 3.87 & -1.331 & $\mathrm{X}$ & KB & 6.08 & $\cdots$ \\
\hline Ca II: & & & & \multicolumn{3}{|c|}{$\log \varepsilon_{\mathrm{LTE}}=6.27 \pm 0.17(9)$} & 4252.632 & 3.84 & -2.018 & $\mathrm{X}$ & $\mathrm{KB}$ & 6.05 & $\cdots$ \\
\hline 4799.973 & 8.44 & -0.419 & $\mathrm{C}$ & $\mathrm{OP}$ & 6.26 & $\ldots$ & 4261.913 & 3.87 & -1.531 & $X$ & $\mathrm{~KB}$ & 5.93 & $\ldots$ \\
\hline 5019.971 & 7.51 & -0.28 & $\mathrm{D}$ & NIST & 6.28 & $\ldots$ & 4275.567 & 3.86 & -1.709 & $X$ & $\mathrm{~KB}$ & 6.01 & $\ldots$ \\
\hline 5307.224 & 7.51 & -0.90 & $\mathrm{D}$ & NIST & 6.36 & $\ldots$ & 4284.188 & 3.86 & -1.864 & $\mathrm{X}$ & KB & 6.02 & $\ldots$ \\
\hline 8248.80 & 7.51 & 0.57 & $\mathrm{C}$ & NIST & 5.95 & $\ldots$ & 4588.199 & 4.07 & -0.63 & D & MFW & 6.05 & $\cdots$ \\
\hline 8254.72 & 7.51 & -0.39 & $\mathrm{C}$ & NIST & 6.04 & $\ldots$ & 4592.049 & 4.07 & -1.22 & $\mathrm{D}$ & MFW & 5.85 & $\ldots$ \\
\hline 8498.02 & 1.69 & -1.47 & $\mathrm{C}$ & OP & 6.43 & $\ldots$ & 4616.629 & 4.07 & -1.29 & $\mathrm{D}$ & MFW & 5.82 & $\ldots$ \\
\hline 8662.14 & 1.69 & -0.77 & $\mathrm{C}$ & OP & 6.39 & $\cdots$ & 4634.070 & 4.07 & -1.24 & D & MFW & 6.12 & $\ldots$ \\
\hline 8912.07 & 7.05 & 0.575 & $X$ & $\mathrm{~KB}$ & 6.41 & $\ldots$ & 4812.337 & 3.86 & -1.99 & $X$ & KP & 5.95 & \\
\hline 8927.36 & 7.05 & 0.750 & $X$ & $\mathrm{~KB}$ & 6.34 & $\ldots$ & 4824.127 & 3.87 & -0.96 & $\mathrm{X}$ & $\mathrm{KP}$ & 6.00 & $\ldots$ \\
\hline Sc II: & & & & \multicolumn{3}{|c|}{$\log \varepsilon_{\mathrm{LTE}}=3.20 \pm 0.10$} & 4836.229 & 3.86 & -1.94 & $\mathrm{X}$ & $\mathrm{KP}$ & 6.01 & \\
\hline 4246.822 & 0.32 & 0.28 & $X$ & KB & 3.24 & & 4848.235 & 3.86 & -1.14 & $X$ & KP & 6.03 & \\
\hline 4324.996 & 0.60 & -0.44 & $\mathrm{~B}$ & MFW & 3.20 & & 4876.399 & 3.86 & -1.46 & $\mathrm{D}$ & MFW & 6.00 & $\cdots$ \\
\hline 5239.813 & 1.46 & -0.77 & $\mathrm{~B}$ & MFW & 3.17 & & 5237.329 & 4.06 & -1.16 & $\mathrm{D}$ & MFW & 5.94 & $\ldots$ \\
\hline 5526.790 & 1.77 & 0.06 & $X$ & $\mathrm{~KB}$ & 3.06 & & 5246.768 & 3.71 & -2.45 & $\mathrm{D}$ & MFW & 5.97 & $\ldots$ \\
\hline 5667.149 & 1.50 & -1.19 & $\begin{array}{l}\mathrm{A} \\
\mathrm{B}\end{array}$ & MFW & 3.33 & $\begin{array}{l}\cdots \\
\ldots\end{array}$ & 5249.437 & 3.76 & -2.426 & $X$ & KB & 5.85 & \\
\hline & & & & & & & 5274.964 & 4.05 & -1.29 & $X$ & $\mathrm{~KB}$ & 5.94 & \\
\hline
\end{tabular}


Table A.1. continued.

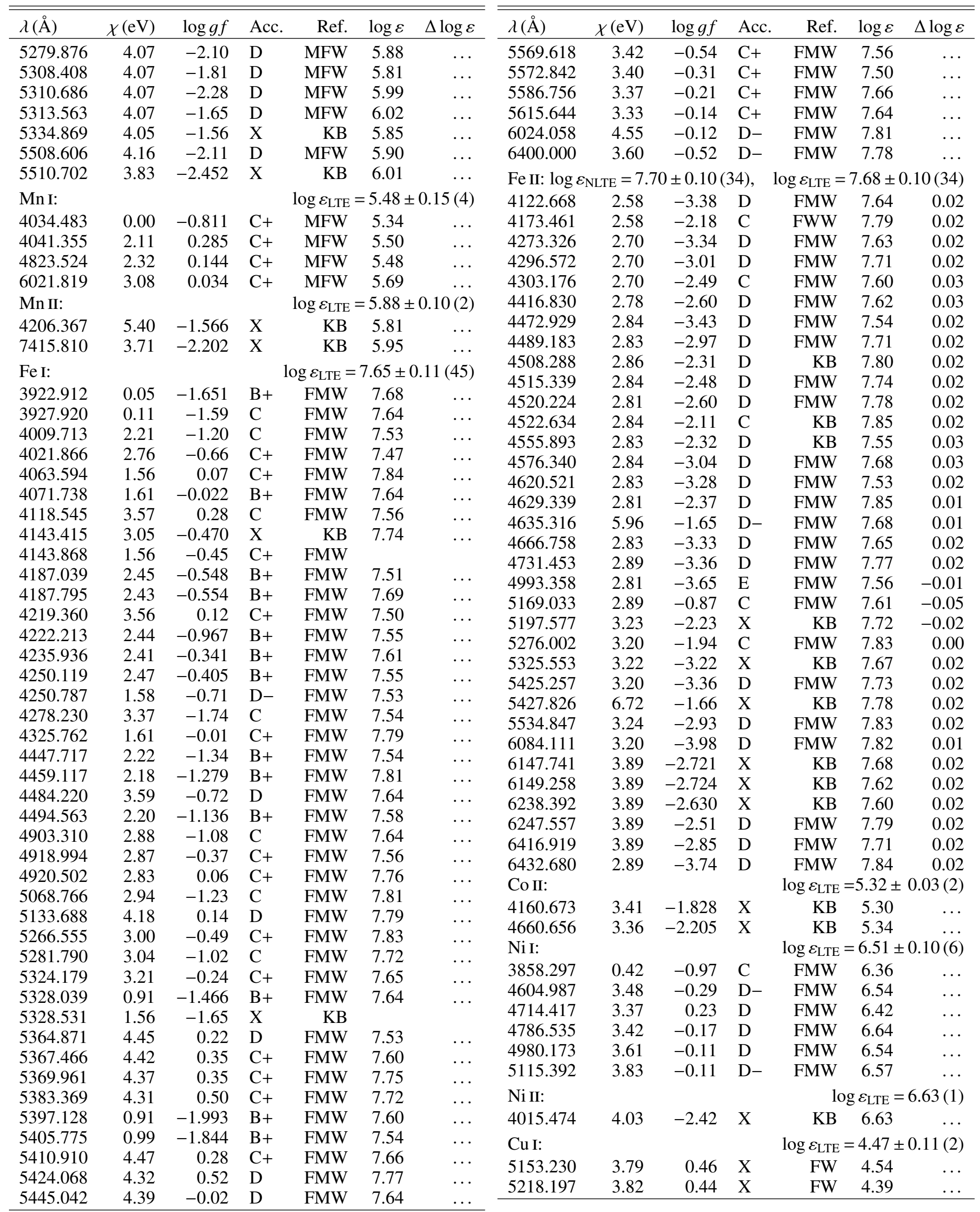


Table A.1. continued.

\begin{tabular}{|c|c|c|c|c|c|c|}
\hline$\lambda(\AA)$ & $\chi(\mathrm{eV})$ & $\log g f$ & Acc. & Ref. & $\log \varepsilon$ & $\Delta \log \varepsilon$ \\
\hline Zn I: & & & \multicolumn{4}{|c|}{$\log \varepsilon_{\mathrm{LTE}}=5.16 \pm 0.06(2)$} \\
\hline 4722.153 & 4.03 & -0.37 & $X$ & $\mathrm{KSZ}$ & 5.20 & \\
\hline 4810.528 & 4.06 & -0.15 & $X$ & $\mathrm{KSZ}$ & 5.12 & $\ldots$ \\
\hline Sr II: & & & \multicolumn{4}{|c|}{$\log \varepsilon_{\mathrm{LTE}}=3.18 \pm 0.03(2)$} \\
\hline 4077.709 & 0.00 & 0.15 & $X$ & FW & 3.16 & \\
\hline 4215.519 & 0.00 & -0.17 & $X$ & FW & 3.20 & $\ldots$ \\
\hline Y II: & & & \multicolumn{4}{|c|}{$\log \varepsilon_{\mathrm{LTE}}=2.14 \pm 0.23(3)$} \\
\hline 3774.339 & 0.13 & 0.22 & $\mathrm{X}$ & $\mathrm{H}$ & 2.40 & \\
\hline 3788.697 & 0.10 & -0.06 & $\mathrm{X}$ & $\mathrm{H}$ & 2.05 & \\
\hline 3950.356 & 0.10 & -0.49 & $X$ & $\mathrm{H}$ & 1.98 & $\ldots$ \\
\hline Zr II: & & & \multicolumn{4}{|c|}{$\log \varepsilon_{\mathrm{LTE}}=3.05 . \pm 0.08$} \\
\hline 4050.329 & 0.71 & -0.99 & $X$ & $\mathrm{~B}$ & 2.98 & \\
\hline 4149.202 & 0.80 & -0.03 & $\mathrm{X}$ & $\mathrm{B}$ & 3.13 & $\ldots$ \\
\hline 4496.974 & 0.71 & -0.81 & $\mathrm{X}$ & $\mathrm{B}$ & 3.05 & $\ldots$ \\
\hline Ba II: & & & \multicolumn{4}{|c|}{$\log \varepsilon_{\mathrm{LTE}}=3.00 \pm 0.18(5)$} \\
\hline 4554.029 & 0.00 & 0.14 & $\mathrm{X}$ & $\mathrm{D}$ & 3.21 & $\ldots$ \\
\hline 4934.076 & 0.00 & -0.16 & $X$ & $\mathrm{D}$ & 3.15 & $\ldots$ \\
\hline 5853.668 & 0.60 & -0.51 & $X$ & $\mathrm{D}$ & 2.77 & $\ldots$ \\
\hline 6141.713 & 0.70 & 0.19 & $X$ & $\mathrm{D}$ & 2.89 & $\ldots$ \\
\hline 6496.897 & 0.60 & -0.01 & $\mathrm{X}$ & $\mathrm{D}$ & 3.00 & $\ldots$ \\
\hline
\end{tabular}

